\title{
移动用户群体聚集行为模型及其高能效资源配置 方法
}

\author{
杨坤 ${ }^{1}$ ，张兴 ${ }^{*}$ ，杨居沃 ${ }^{1}$ ，王硕 ${ }^{1}$ ，梅承力 ${ }^{2}$ ，王文博 ${ }^{1}$
}

1. 北京邮电大学泛网无线通信教育部重点实验室, 北京 100876

2. 中国电信股份有限公司技术创新中心, 北京 100032

* 通信作者. E-mail: hszhang@bupt.edu.cn

收稿日期: 2017-02-16; 接受日期: 2017-03-23; 网络出版日期: 2017-05-08 国家自然科学基金 (批准号: 61372114, 61631005)、国家重点基础研究计划 (973) (批准号: 2012CB316005) 和北京市科技新星计 划 (批准号: Z151100000315077) 资助项目

摘要 由于用户社会属性的存在, 复杂蜂窝移动网络的业务特征和用户行为在时域、空域和内容等 多维度上的分布都呈现出以群体为特征的聚集行为规律. 以往静态、孤岛式的网络资源配置方法造 成了网络资源的巨大浪费, 因此利用用户群体行为特征规律将存在巨大的能效和资源利用提升空间. 基于对实际运营的蜂窝移动通信系统中数据的采集和测量, 首先从空间、时间等多个维度对用户群 体聚集行为进行了深入分析研究, 得到了基站流量在空域、时域和空 - 时联合的分布规律. 研究表 明，业务在空间符合 Log-normal 分布，其参数与典型区域类型有关; 用户数及其产生的业务量随着 时间变化具有明显的规律性, 正弦叠加模型能够很好地反映出现网实际业务量的变化情况. 其次, 通 过对空域和时域的联合分析, 得到了能精准预测基站业务变化的空 - 时联合分布模型. 与实际数据 对比发现, 该模型准确度可以达到 $93 \%$ 以上. 为了更明确地表征用户群体聚集行为, 利用经济学中 的基尼系数对用户群体聚集行为进行了数学定义和定量描述. 最后, 基于所提出的业务空 - 时模型 和用户群体行为聚集模型, 提出了几种高能效的无线网络资源配置方法、传输控制方法和基站分级 休眠策略, 探索利用用户群体行为规律提升无线网络能效的新途径.

关键词用户群体聚集行为, 资源分配, 能量效率, 移动蜂窝网络

\section{1 引言}

蜂窝无线网络中, 用户在时间、空间及业务内容等多个维度呈现明显的群体行为规律, 相对于个 体行为, 用户的群体聚集行为的研究分析将更有助于无线网络的架构设计、资源优化和服务控制. 业

\footnotetext{
引用格式: 杨坤, 张兴, 杨居沃, 等. 移动用户群体聚集行为模型及其高能效资源配置方法. 中国科学: 信息科学, 2017, 47: 620-636, doi: 10.1360/N112017-00043

Yang $\mathrm{K}$, Zhang $\mathrm{X}$, Yang $\mathrm{J} \mathrm{W}$, et al. The model of large-scale mobile user behavior and energy efficiency resource allocation method (in Chinese). Sci Sin Inform, 2017, 47: 620-636, doi: 10.1360/N112017-00043
} 
务的特征规律是用户行为研究中的重要部分, 近年来学术界对无线网络中的业务分布规律有非常广泛 和深入的研究. 文献 [1] 给出了业务在时间维度上缩放不变性, 又叫做自相似性, 即观察任意一段时间 内到达的业务, 所得到的规律与观察所有时间内得到的总体规律一致. 更进一步, 如果业务在相当长的 尺度内都存在自相似性, 这种现象叫做业务的长相关性 ${ }^{[2]}$. 业务的不均匀性在业务概率分布上的体现 就是其分布函数不是均匀分布. 长尾概率分布是描述业务不均匀性的一类经典分布, 长尾分布是指业 务的分布函数以自变量的负数次幂速率衰减, 这种衰减速度慢于 Poisson 分布等非长尾分布的衰减速 度. Weibull 和 Pareto 分布是两种经典的长尾分布, 文献 [3] 中作者分析了 10 种不同类型的业务在时 间维度上的分布规律, 首次提出用 Weibull 分布来刻画业务的分布, 其中 Weibull 分布的形状参数小 于 0.65 则意味着业务的分布是长尾分布. 文献 [4] 中, 作者进一步研究了不同类型的业务在到达时间 上的分布, 并给出相应 Weibull 分布两个参数的取值范围. 长尾分布可以存在随机变量方差无穷的情 况, 文献 [5] 研究了业务的统计数据, 发现 Pareto 分布正好可以通过参数的调整分别表征有限和无限 方差两种情况.

在业务的空间分布规律方面, 文献 [6] 的作者把一个基站的覆盖范围划分成不同的区域, 用每个区 域内的业务量与总业务量的百分比来刻画这个区域业务量的大小; 然后计算不同区域的中断概率以及 误块率, 来为每个区域配置合适的发射功率. 文献 [7] 分析了实际蜂窝网络中不同区域内业务量的统计 结果, 认为业务量在空间上的分布服从对数正态分布. 在此基础上, 文献 [8] 的作者进一步给出了一整 套生成整个区域内业务量的方法, 所生成的业务量在空间上服从对数正态分布, 并且能够体现出业务 的空间相关性. 该作者在后续研究 ${ }^{[9]}$ 中, 设计出一套无线网络不同类型基站的部署策略, 以适应这种 具有空间相关性的业务分布. 在另外一篇研究业务空间分布的文章 [10] 中, 作者使用业务需求点的概 念描述业务的空间不均匀性. 首先对空间进行大致划分, 然后对每个划分出来的区域进行第二次划分, 每一次划分的过程都以面积相等为准则来划分, 但是如果某区域内的业务量小于某个门限值, 那对该 区域的划分就停止. 因此业务量越高的区域, 划分出的小方格个数越多, 面积越小. 划分完成后, 所有 这些区域的中心都定义成业务量需求点. 作者通过实际数据的测量, 得到在固定面积区域内的业务量 需求点个数是服从对数正态分布的.

在高能效资源配置和传输控制策略方面, 近年来, 学术界对把低功耗的微基站引入无线网络的优 缺点作了非常深入的研究 ${ }^{[11 ~ 14]}$ : 引入小基站会增加对无线网络其他资源的占用, 例如在空间上占用 宏基站的站址资源, 在相同频率资源上有可能与已经存在的宏基站形成互相干扰, 使边缘用户的传输 质量发生恶化. 因此要发挥微基站的低功耗优点, 就必须合理部署微基站并为其有效分配无线资源. 从功率分配的角度看, 功率的大小变化意味着覆盖范围的变化以及覆盖范围内用户传输速率的变化. 如果分配给微基站的功率为零, 就意味着微基站处于休眠状态. 文献 [15] 中, 作者对分配给宏基站和 微基站的资源进行划分, 使之互相不存在干扰, 并推导了最优的资源划分方式, 但是这种资源分配的 方式会导致频谱效率变低. 文献 [16] 中, 作者采用了复用频率资源的方法来提高无线网络的频谱效率, 但缺点是将导致边缘用户收到的干扰加剧. 所以在一定程度上抵消了频谱效率的提升, 从而导致能量 效率下降. 由此可见, 在复用频谱源的场景下, 宏基站和微基站的干扰抑制尤为重要. 为了抑制基站间 干扰, 文献 [17] 从用户接入控制的角度出发, 规定如果用户的接入会使得服务基站需要增加大量的发 射功率导致基站间干扰加剧, 就不让该用户接入这个基站. 文献 $[18,19]$ 从控制功率减少干扰的角度出 发, 适当降低宏基站或者微基站的发射功率, 虽然这样功率分配会使得本小区用户的频谱效率有所下 降, 但是本基站也会对邻小区用户干扰. 因此通过适当降低无线网络的频谱效率, 提高整个网络的能 量效率.

当前研究中对业务的不均匀性缺乏一个统一的建模方式, 以及没有有效的衡量指标, 不能够对业 


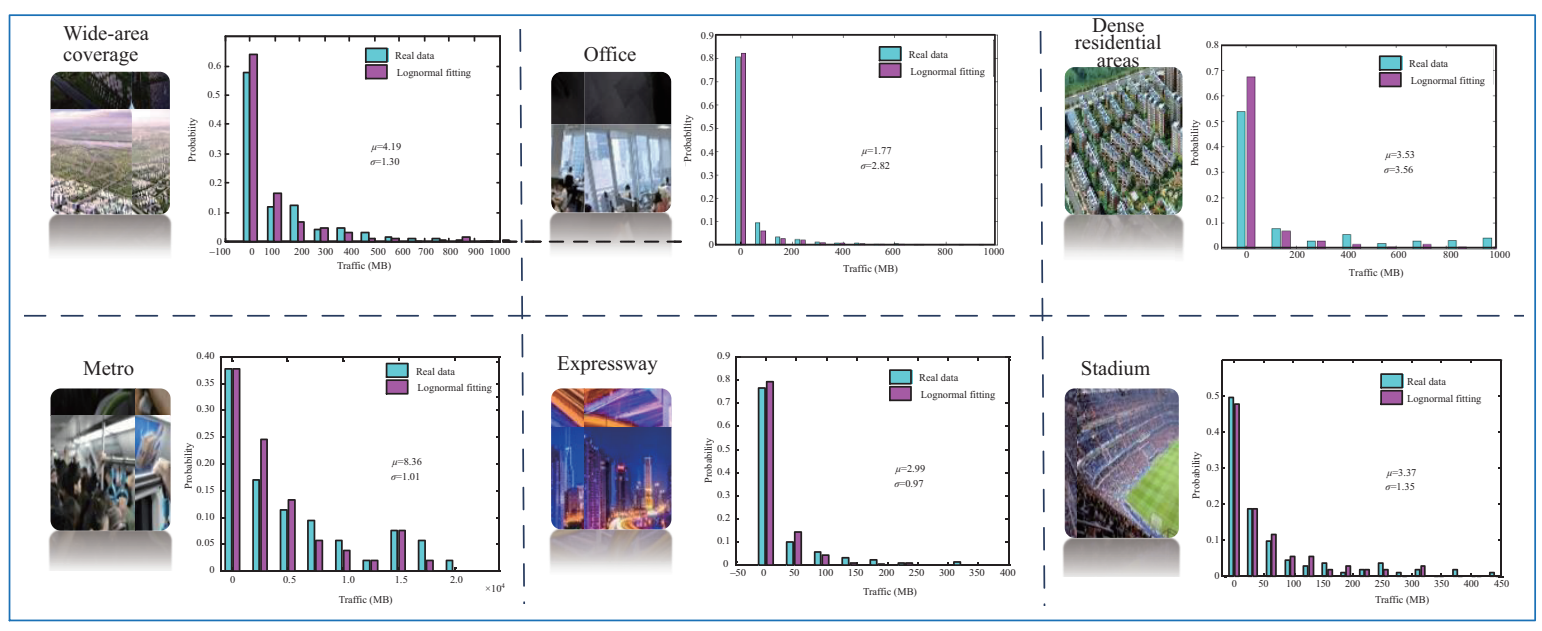

图 1 (网络版彩图) 典型场景业务空间分布模型

Figure 1 (Color online) Space domain model of typical traffic

务各个维度上的不均匀性进行定量描述. 其次在聚集行为特征研究和应用方面, 特别是对多种基站共 存的异构网络的聚集特性也不能够定量地描述. 因此需要对时间、空间及业务内容等多个维度的群体 行为规律进行分析, 以得到基站流量在时域、空域和空 - 时联合的分布规律, 以及用户群体行为模型. 同时利用用户群体聚集行为规律得到业务聚集特性的表征方法, 并利用该表征方法对蜂窝网络进行分 析, 为蜂窝网络架构设计、资源配置和服务控制的研究奠定模型基础.

\section{2 用户群体聚集行为模型}

\section{1 空间维度聚集模型}

受地理环境的影响, 蜂窝网络中不同区域的用户会表现出不同的行为特点, 从而导致流量在空间 维度上的不均匀分布. 业务流量在空间上的分布能够一定程度上反映出用户行为特征, 所以流量空间 建模对优化网络架构、提高蜂窝网络的服务质量等具有重要的意义. 已有研究 ${ }^{[8]}$ 表明蜂窝网络业务的 空间不均匀性可以用对数正态 (Log-normal) 分布来建模. Log-normal 分布的概率密度函数为

$$
f_{X}(x ; \mu, \sigma)=\frac{1}{x \sigma \sqrt{2 \pi}} \mathrm{e}^{\frac{(\ln x-\mu)^{2}}{2 \sigma^{2}}},
$$

其中 $\mu$ 与 $\sigma$ 分别是变量对数的平均值和标准差.

由于网络中用户的行为不同, 基站和业务量的分布并不完全统一. 因此, 现实中存在很多典型场 景区域. 本文从 IMT-2020 (5G) 所建议的蜂窝网络典型场景中, 选择了广域覆盖、办公区、密集宅、地 铁、高速公路和体育场等 6 大场景, 对其业务流量空间分布进行了深入分析并给出了其典型值, 结果 如图 1 所示.

从图 1 中的结果可以得到以下规律, 即 IMT-2020 (5G) 典型场景中用户业务流量在空域近似符合 Log-normal 分布, 各场景的分布均值与方差不尽相同, 与场景密切相关.

本研究相关工作发表在文献 $[20,21]$ 上. 

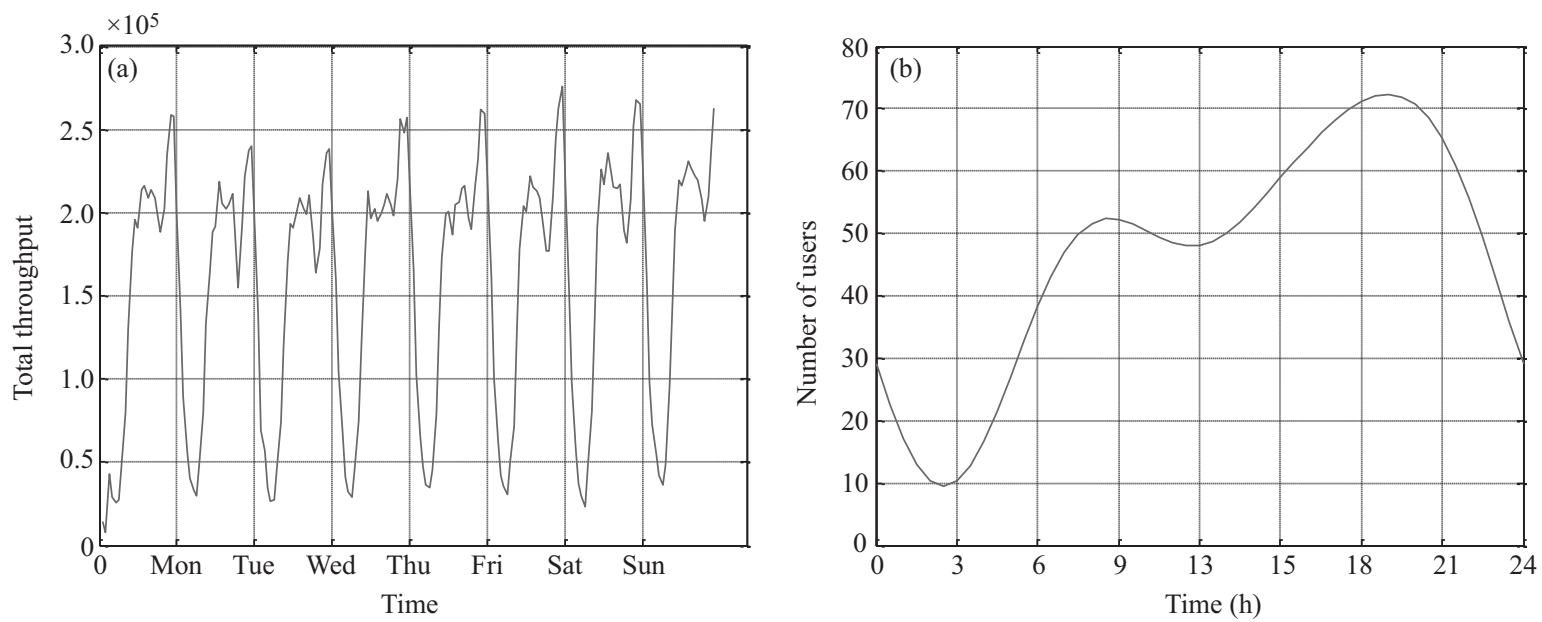

图 2 （a）实际网络一周内业务量变化情况; (b) 每天业务量变化情况

Figure 2 (a) The change of one week traffic in real network; (b) the change of one day traffic

\section{2 时间维度聚集模型}

当前无线通信业务变化规律比较复杂, 传统的单正弦模型已经无法刻画出用户群体在多个维度上 的行为, 例如上班业务高峰、休闲业务高峰和工作日高峰等, 因此需要更加全面细致地对业务时间变 化规律进行建模分析. 针对不同地区、不同业务请求的业务量进行建模, 充分挖掘不同业务之间的区 别与联系, 更加精确完整地反映出实际网络的规律. 在时域建模的第一阶段工作中, 通过采集得到实测 基站的 DPI (deep packet inspection) 数据进行分析, 选择南京地区 2012 年 6 月 5 日到 7 月 5 日一个 月的数据 (未区分业务种类) 来进行分析, 数据记录的时间颗粒度为半个小时, 分析结果如图 2 所示.

由于每天业务量的规律呈现出明显的周期性, 而且常用的理论分析模型 (理想正弦模型及类正弦 模型) 均无法准确地表述出上述曲线的双峰值特性, 因此, 对这一天的数据进行了快速 Fourier 变换, 提取出主要的频点进行拟合. 如图 3 所示, 业务量变化具有明显的多周期特性, 因此, 在建模的过程中 需要保证选取的频点使得确定系数 (R-square) 在 0.8 以上. 最终拟合得到的业务量表达式如下:

$$
\rho_{t}=a_{0}+a_{1} \sin \left(\frac{\pi t}{12}+\varphi_{1}\right)+a_{2} \sin \left(\frac{\pi t}{6}+\varphi_{2}\right)+a_{3} \sin \left(\frac{\pi t}{4}+\varphi_{3}\right),
$$

其中, $\rho_{t}$ 代表拟合的业务量, $a_{0}$ 为常数, 代表业务量的平均值, $a_{i}$ 和 $\varphi_{i}$ 分别代表其对应的幅值及相位 值. 最后可以将拟合得到的正弦叠加模型与常用的理想正弦模型及类正弦模型对实际数据的拟合情况 进行对比.

为了验证所提模型的准确性, 选择中国不同城市、不同网络制式下的基站业务流量数据, 并使用 所提出的正弦波叠加模型进行数据分析和拟合, 拟合结果如图 4 所示. 通过所提正弦波叠加模型对基 站流量时域拟合图, 证明了所提拟合方法的准确性. 图 5 分别给出了南京、香港和苏州在不同网络类 型下的基站流量时域模型. 测试证明, 模型准确度达到 $90 \%$ 以上.

通过对实际网络中的业务量时间变化规律进行建模和分析, 并且针对不同的地区和不同网络类型, 对用户数目、流量大小等内容进行分析. 从这些分析中发现, 正弦叠加模型能够很好地反映出实际业 务量的变化情况. 同时, 从式 (2) 以及不同城市不同网络的结果来看, 24,12 和 8 小时这 3 种周期在时 域变化中普遍存在, 这 3 种周期恰好对应天、白天 (晚上) 和工作时间. 


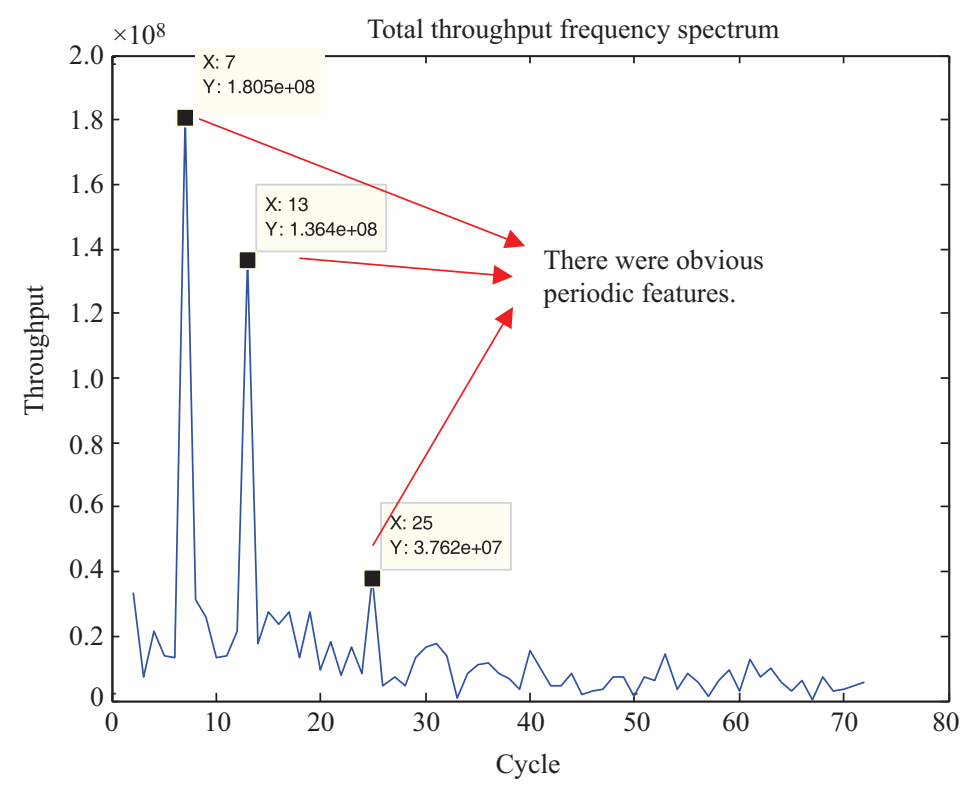

图 3 对实际网络中一天的业务量进行 FFT 变化

Figure 3 One day traffic in real network after FFT transform
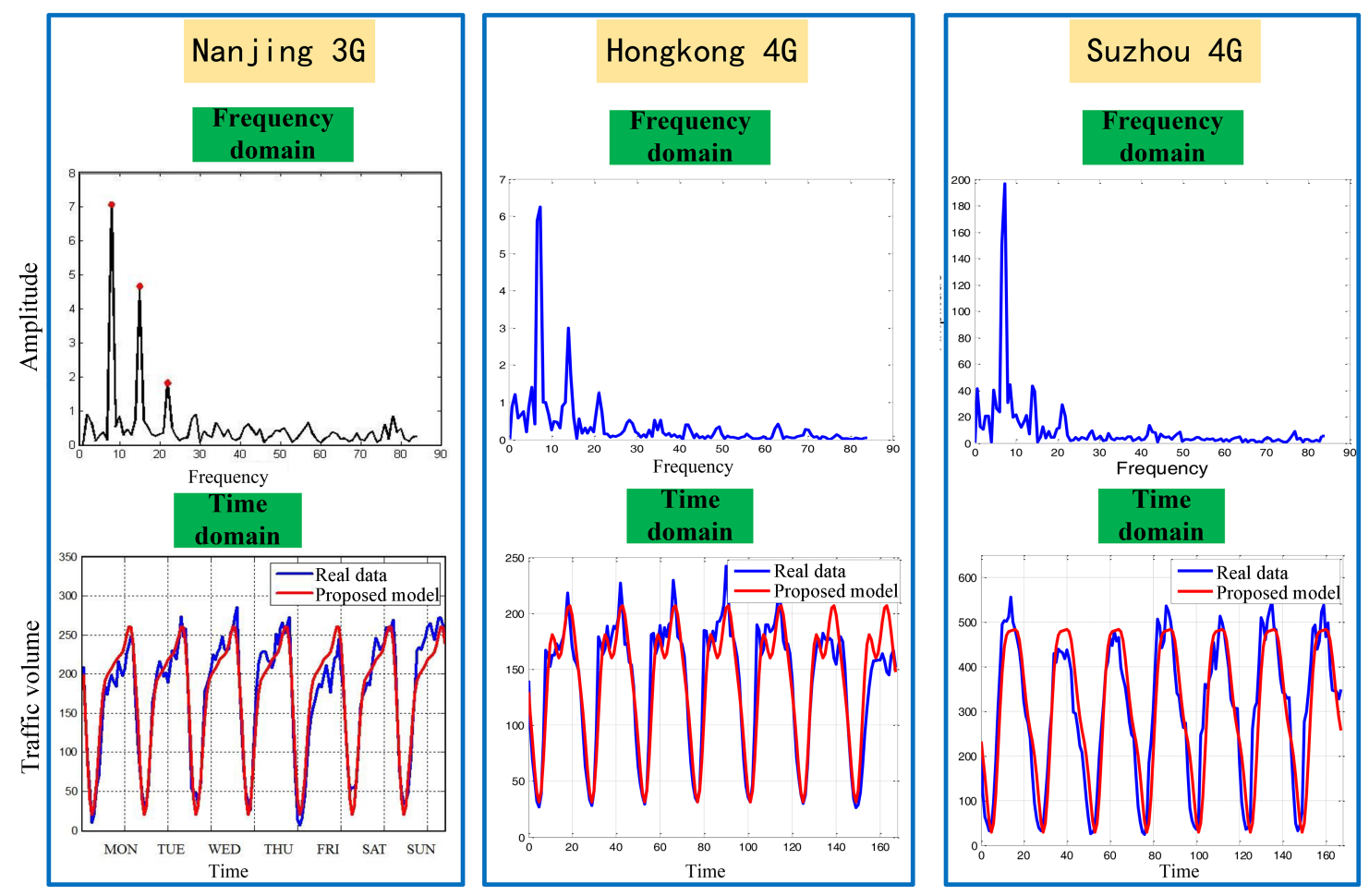

图 4 不同城市不同网络制式下基站流量频域分析及时域拟合图

Figure 4 The frequency domain analysis and time domain fitting of traffic in different cities and networks 


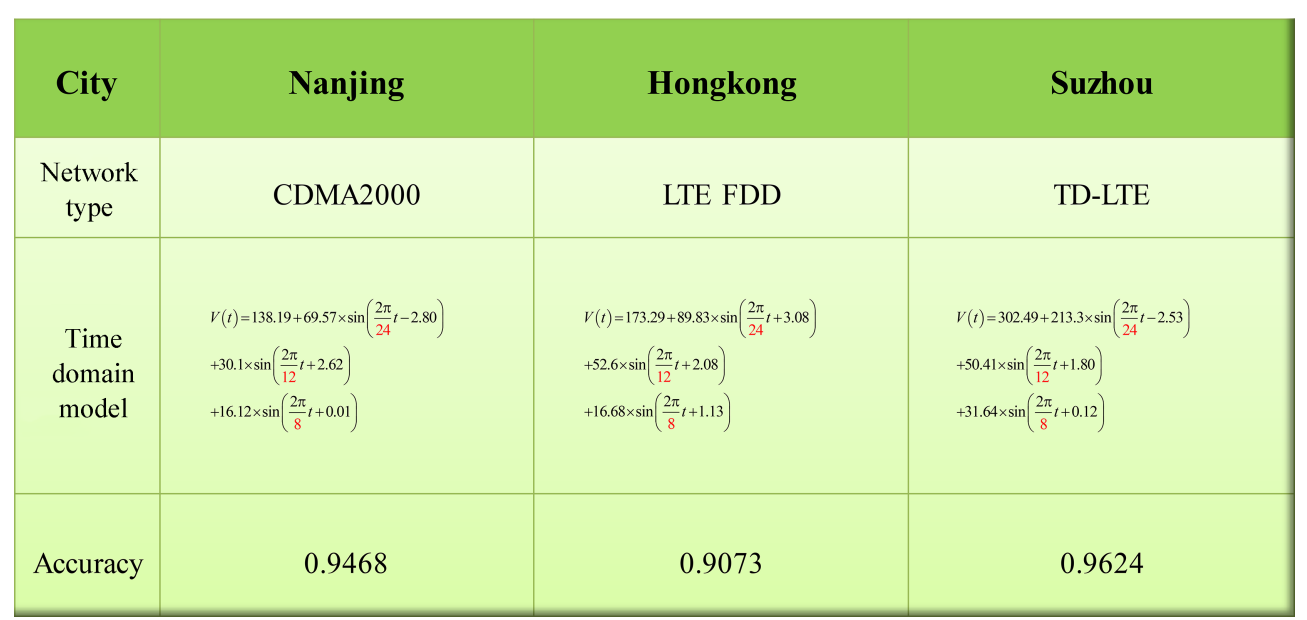

图 5 (网络版彩图) 不同城市不同网络制式下基站流量时域分布模型及准确度

Figure 5 (Color online) The time domain model and accuracy of traffic in different cities and networks

\section{3 空 - 时联合分布模型}

从基站的空域和时域分析中, 发现多个基站业务流量叠加之后在时域具有明显的周期性, 也即一 定区域范围内基站叠加后才具备明显周期规律，而单个基站流量在时域上的变化没有明确的周期性. 单个基站在一周之内的流量变化情况呈现随机性, 没有明显的规律. 传统模型不能反映这种随机性, 因 此其准确性不高.

为了能更好地描述单基站的流量变化情况, 提出一种空 - 时联合建模方法. 该分析方法能够精确 建模基站模式下的流量变化情况.

基站流量空 - 时联合分布模型建模过程如下:

(1) 针对典型区域, 利用正弦波叠加模型计算 $t$ 时刻的平均流量:

$$
m(t)=\frac{1}{N}\left(a_{0}+\sum_{k=1}^{3} a_{k} \sin \left(2 \pi \frac{k t}{24}+\varphi_{k}\right)\right) ;
$$

(2) 取典型区域的经验值参数 $\sigma$, 计算模型参数 $\mu(t)$ :

$$
\mu(t)=\log [m(t)]-\frac{1}{2} \sigma^{2} ;
$$

(3) 得到基站 $i$ 在时刻 $t$ 的流量 $V_{i}(t)$ :

$$
V_{i}(t)=\frac{1}{t \sigma \sqrt{2 \pi}} \exp \left\{-\frac{[\ln t-\mu(t)]^{2}}{2 \sigma^{2}}\right\} .
$$

为了验证空 - 时模型的准确性, 对比公园、商业区和学校的实际流量与模型产生的流量之间的差 异. 如图 6 所示, 实线为实际流量数据, 圆点标记实线为模型产生的流量数据. 通过对比发现, 所提空 - 时模型的准确性达到 $93 \%$ 以上. 因此, 所提模型可以对实际蜂窝网络时域和空域进行准确建模.

本研究相关工作发表在文献 [21] 上. 

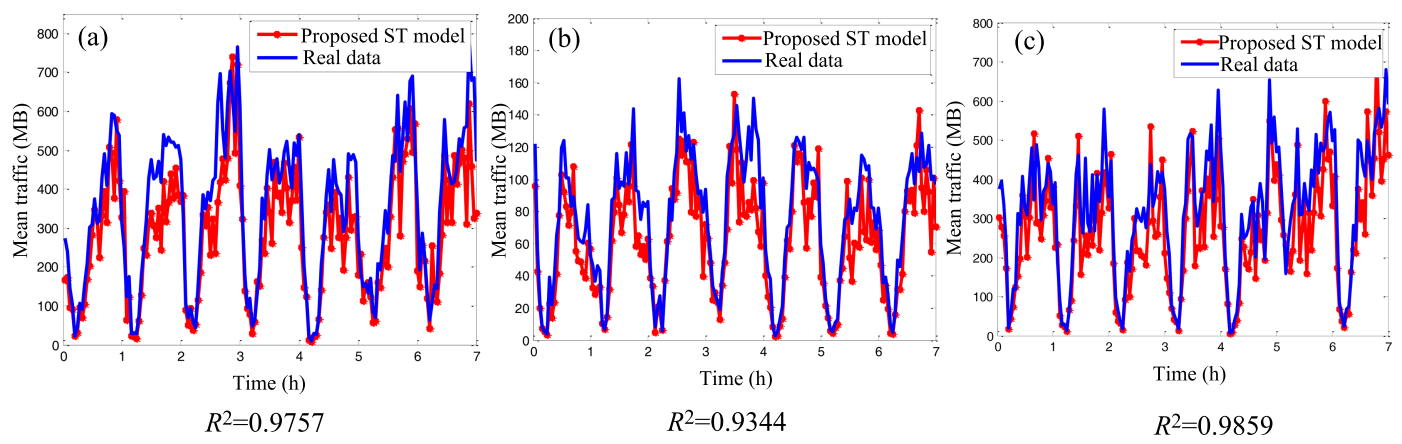

图 6 (网络版彩图) 不同区域实际数据与模型预测对比

Figure 6 (Color online) The comparison of real data and model in different areas. (a) Park; (b) business zone; (c) school

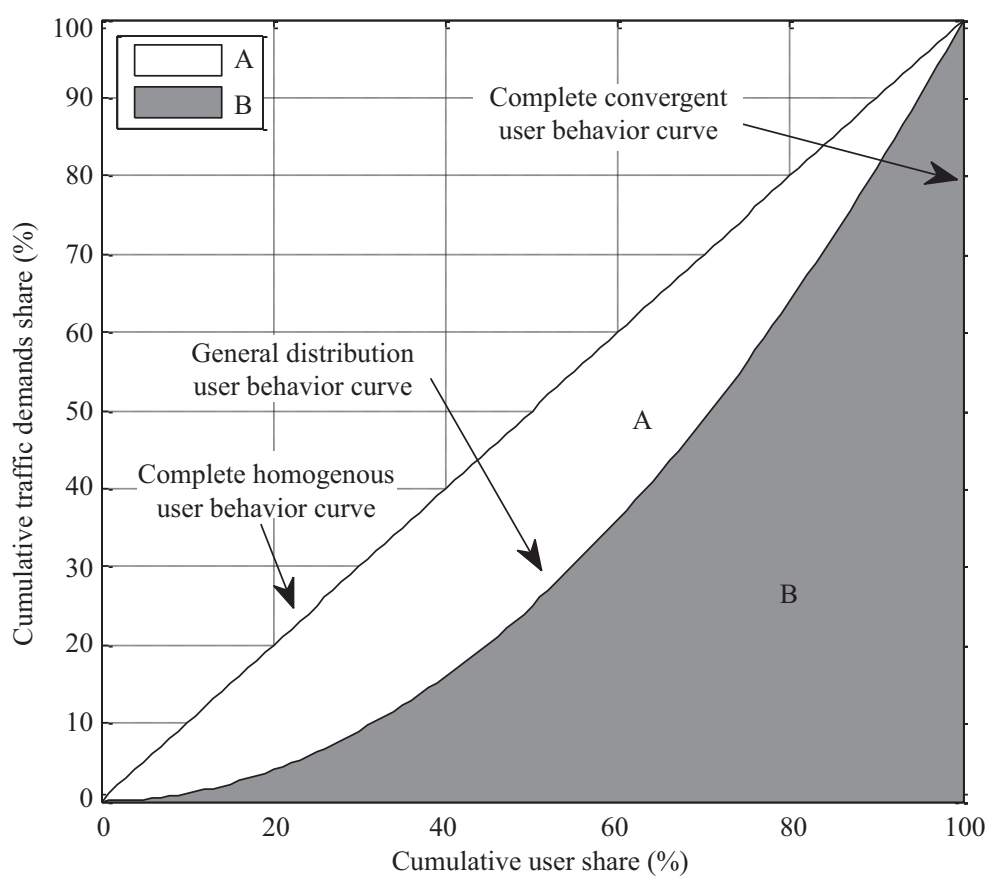

图 7 业务聚集系数定义示意图

Figure 7 The definition of traffic convergence coefficient

\section{4 基于基尼系数的聚集行为表征方法}

为了描述用户群体行为的物理意义, 对用户群体行为建立如下定义: 用户在网络中以群体为单位, 在活动规律、业务需求、接入频率和聚集特性等多维度下的行为模式和特征规律. 为了更明确表征用 户群体行为, 使用经济学中的基尼系数对用户群体行为进行定量描述, 如图 7 所示.

用户聚集系数表达式为

$$
h=\frac{A}{A+B},
$$

其中 $A$ 和 $B$ 分别代表图 7 中面积的大小.

图 7 中业务量分布曲线越上凸意味着聚集程度越低; 当业务量分布曲线与完全均匀分布业务量曲 626 
线重合, 意味着用户完全没有聚集性, $h=0$; 业务量分布曲线越下凸意味着聚集程度高; 当业务量和集 中业务量分布曲线重合, 意味着用户完全聚集, $h=1$.

通过用户聚集系数的表达, 可以得到几种常见分布的 $h$ 表达式.

当业务分布为对数正态分布时, 聚集系数表达式为

$$
h=\operatorname{erf}\left(\frac{1}{2} \sqrt{\ln \left(1+(\sigma / \mu)^{2}\right)}\right),
$$

其中 $\mu$ 与 $\sigma$ 分别是变量对数的平均值和标准差.

当业务分布为指数分布时, 聚集系数表达式为

$$
h=1-\lambda \int_{0}^{\infty} \mathrm{e}^{-2 \lambda x} \mathrm{~d} x=1 / 2 .
$$

当业务分布为均匀分布时, 聚集系数表达式为

$$
h=\frac{\sqrt{3}}{3} \frac{\sigma}{\mu}, \quad \frac{\sigma}{\mu}<\frac{\sqrt{3}}{3},
$$

其中 $\mu$ 与 $\sigma$ 分别是均匀分布随机变量的均值和方差.

当业务分布为 Gamma 分布时, 聚集系数表达式为

$$
h=1-\frac{1}{\left(\frac{\mu^{2}}{\sigma^{2}}\right)} \cdot \frac{\left(2 \frac{\mu^{2}}{\sigma^{2}}\right)}{\left(\frac{\mu^{2}}{\sigma^{2}}+1\right) 2^{2 k-1}} 2 F_{1}\left(1,2 \frac{\mu^{2}}{\sigma^{2}}+1 ; \frac{\mu^{2}}{\sigma^{2}}+2,1 / 2\right),
$$

其中 $\mu$ 与 $\sigma$ 分别为 Gamma 分布随机变量的均值和方差, ${ }_{2} F_{1}(\cdot)$ 为超几何函数.

接下来, 对异构分层蜂窝网络的聚集特性进行描述. 蜂窝网络理论分析常以空间 Poisson 点过程 (PPP) 作为理论工具, 整个无线接入网分成两层: 宏基站为第 1 层, 基站位置服从密度 $\lambda_{M}$ 的 Poisson 分布, 宏基站发射功率为 $P_{M}$; 微基站为第 2 层, 基站位置服从密度 $\lambda_{m}$ 的 Poisson 分布, 微基站发射 功率为 $P_{m}$.

假设用户非均匀分布, 热点区域用户密度为 $\lambda_{m}^{\mathrm{u}}$, 热点用户聚集度高, 请求的业务量大, 用 $r_{m}^{\mathrm{u}}$ 表示 热点用户的业务量; 宏基站区域用户密度为 $\lambda_{M}^{\mathrm{u}}$, 宏基站用户聚集度低, 请求的业务量小, 用 $r_{M}^{\mathrm{u}}$ 表示 非热点用户的业务量. 另外, 根据随机几何理论可以计算出宏基站覆盖区域和微基站覆盖区域占全部 区域的比例分别为

$$
\begin{aligned}
\gamma_{M} & =\frac{\lambda_{M} P_{M}{ }^{2 / \alpha}}{\lambda_{m} P_{m}{ }^{2 / \alpha}+\lambda_{M} P_{M}{ }^{2 / \alpha}}, \\
\gamma_{m} & =\frac{\lambda_{m} P_{m}{ }^{2 / \alpha}}{\lambda_{m} P_{m}{ }^{2 / \alpha}+\lambda_{M} P_{M}{ }^{2 / \alpha}} .
\end{aligned}
$$

可以算出在这个模型里面的用户业务的总量为

$$
R=\lambda_{M}^{\mathrm{u}} r_{M}^{\mathrm{u}} \gamma_{M}+\lambda_{m}^{\mathrm{u}} r_{m}^{\mathrm{u}} \gamma_{m}
$$

那么所有宏基站用户占业务的比例为

$$
\nu_{M}=\frac{\lambda_{M}^{\mathrm{u}} r_{M}^{\mathrm{u}} \gamma_{M}}{\lambda_{M}^{\mathrm{u}} r_{M}^{\mathrm{u}} \gamma_{M}+\lambda_{m}^{\mathrm{u}} r_{m}^{\mathrm{u}} \gamma_{m}} .
$$


根据前述用户聚集系数的定义, 可以计算出用户业务聚集系数表达式:

$$
\begin{aligned}
h & =1-\gamma_{M} \nu_{M}-\left(1+\nu_{M}\right)\left(1-\gamma_{M}\right)=\left|\frac{\lambda_{M}^{\mathrm{u}} r_{M}^{\mathrm{u}} \gamma_{M}}{\lambda_{M}^{\mathrm{u}} r_{M}^{\mathrm{u}} \gamma_{M}+\lambda_{m}^{\mathrm{u}} r_{m}^{\mathrm{u}} \gamma_{m}}-\gamma_{M}\right| \\
& =\left|\frac{\lambda_{m}^{\mathrm{u}} r_{m}^{\mathrm{u}} \gamma_{m}}{\lambda_{M}^{\mathrm{u}} r_{M}^{\mathrm{u}} \gamma_{M}+\lambda_{m}^{\mathrm{u}} r_{m}^{\mathrm{u}} \gamma_{m}}-\gamma_{m}\right| .
\end{aligned}
$$

对式 (15) 进一步分析, 可分为两种情况:

(1) 当热点区域用户的业务量比宏基站用户的业务量大很多的时候, 用户业务聚集系数为

$$
h=\gamma_{M}=1-\gamma_{m}, \quad r_{m}^{\mathrm{u}}>>r_{M}^{\mathrm{u}},
$$

即用户业务聚集系数由宏基站覆盖区域和微基站覆盖区域占全部区域的比例决定. 所以当热点用户的 业务量比宏基站用户的业务量大很多, 而且微基站覆盖区域占全部区域比例很小的时候, 聚集系数为 1 , 也就是该网络中极少的用户请求了极大的业务量.

(2) 当热点用户的业务量比宏基站用户的业务量小很多的时候, 用户业务聚集系数为

$$
h=1-\gamma_{M}=\gamma_{m}, \quad r_{M}^{\mathrm{u}}>>r_{m}^{\mathrm{u}},
$$

即用户业务聚集系数由宏基站和微基站覆盖区域占全部区域的比例决定. 所以当热点用户的业务量比 宏基站用户的业务量小很多, 而且微基站覆盖区域占全部区域比例很小的时候, 聚集系数为 0 , 即网络 中的业务量是均匀分布在整个区域的.

本研究相关工作发表在文献 [22] 上.

\section{3 高能效资源配置和传输控制方法}

在无线通信网络中存在多种资源, 包括空域资源、功率资源和带宽资源, 而在超蜂窝网络中又多 了一种资源: 不同类型基站的资源. 然而这些资源的传输能力 (容量和覆盖) 和部署成本 (能量消耗和 成本造价) 各不相同, 因此如何将这些资源有效地组合起来充分利用, 使整个无线通信网络的传输能 力能够保证用户需求, 同时尽可能节省能量消耗和降低部署代价, 是研究无线通信网络的基本问题. 众 所周知, 业务的不均匀性为网络资源如何适配业务的需求带来了极大的挑战. 然而, 超蜂窝网络中引 入了低功耗的微基站, 其小范围高容量的传输能力, 为解决业务不均匀性的适配问题提供了可能, 同时 由于其静态功率非常低, 因而为提高整个无线网络的能量效率提供了很好的基础.

\section{1 基于用户群体行为的高能效资源配置方法}

研究业务在热点和非热点两种区域不均匀分布的场景下 (如图 8 所示) 超蜂窝网络的宏基站发射 功率、微基站发射功率, 宏基站密度、微基站密度的控制问题. 要求超蜂窝网络的微基站和宏基站能够 覆盖面积不相等的热点和非热点两种区域, 并满足这两种不同区域的业务速率需求. 本质上来说, 是 通过超蜂窝网络的异构性 (宏基站发射功率、微基站发射功率, 宏基站密度、微基站密度的不同) 来适 配用户业务需求的异质性 (热点区域和非热点区域业务速率和面积的差异), 目标是在确保用户需求的 条件下达到超蜂窝网络能效最优.

在高能效资源配置方法研究中, 描述用户需求有 3 个基本参数: 热点区域单位面积业务速率和非 热点区域单位面积业务速率的比例 $\nu_{m}$ (简称为热点速率比)、热点区域面积和非热点区域面积的比例 $\gamma_{m}$ (简称为热点面积比) 和满足用户 $\operatorname{QoS}$ 所要求的最低信干噪比门限值 $\beta$.

628 


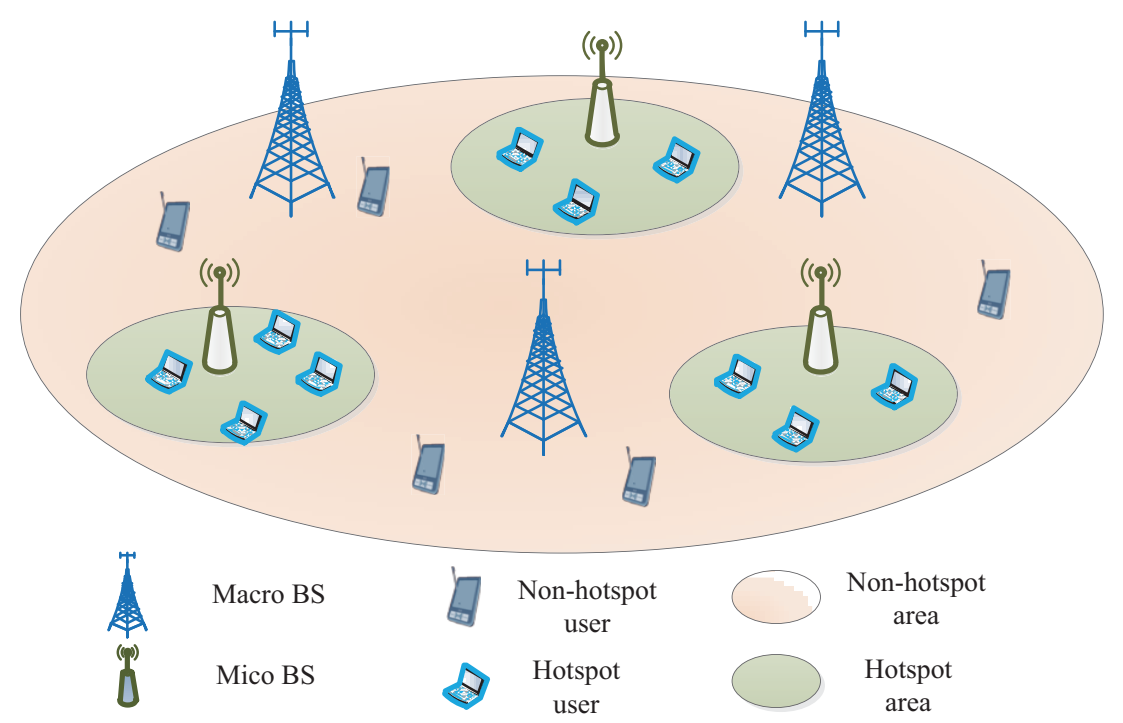

图 8 (网络版彩图) 双层异构网络示意图

Figure 8 (Color online) Two-tier heterogeneous network

在所给定的场景下, 宏基站或微基站可提供的空间平均速率 [16] 可以表达为

$$
\mathrm{ASR}_{k}=D(\alpha, \beta) \frac{\lambda_{k}^{2} P_{k}{ }^{2 / \alpha}}{\lambda_{m} P_{m}^{2 / \alpha}+\lambda_{M} P_{M}^{2 / \alpha}}, \quad k \in\{m, M\},
$$

其中, $D(\alpha, \beta)=\frac{\pi \log (1+\beta)}{C(\alpha) \beta^{2 / \alpha}}+\alpha \pi \frac{2 F_{1}(1,2 / \alpha, 1+2 / \alpha,-1 / \beta)}{2 C(\alpha) \beta^{2 / \alpha}}, C(\alpha)=2 \pi^{2} \csc (2 \pi / \alpha) \alpha^{-1},{ }_{2} F_{1}(\cdot)$ 为超几何函数. 因此, 宏基站和微基站提供的空间平均速率比值 $\widetilde{\nu}_{m}$ (简称为传输速率比) 为

$$
\tilde{\nu}_{m}=\frac{\operatorname{ASR}_{m}}{\operatorname{ASR}_{M}}=\frac{\lambda_{m}^{2} P_{m}^{2 / \alpha}}{\lambda_{M}^{2} P_{M}^{2 / \alpha}} .
$$

在所给的场景下, 宏基站或微基站所能提供的接入成功概率可以表达为

$$
P_{\mathrm{AS}}^{k}=\frac{\pi}{C(\alpha) \beta^{2 / \alpha}} \cdot \frac{\sum_{k=m, M} \lambda_{k} P_{k}^{2 / \alpha}}{\lambda_{m} P_{m}{ }^{2 / \alpha}+\lambda_{M} P_{M}^{2 / \alpha}} .
$$

因此, 宏基站和微基站覆盖面积比值 $\tilde{\gamma}_{m}$ (简称为覆盖面积比) 为

$$
\tilde{\gamma}_{m}=\frac{P_{\mathrm{AS}}^{m}}{P_{\mathrm{AS}}^{M}}=\frac{\lambda_{m} P_{m}{ }^{2 / \alpha}}{\lambda_{M} P_{M}{ }^{2 / \alpha}} .
$$

假设超蜂窝网络的微基站和宏基站能够分别满足覆盖面积不相等的热点和非热点两种区域, 并满 足这两种不同区域的业务速率需求. 因此, 得到业务需求对网络资源配置的传输速率限制和覆盖面积 限制.

(1) 传输速率限制. 微基站和宏基站提供的空间平均速率比值与热点区域单位面积业务速率和非 热点区域单位面积业务速率的比例相等, 即

$$
\nu_{m}=\tilde{\nu}_{m}=\frac{\lambda_{m}^{2} P_{m}{ }^{2 / \alpha}}{\lambda_{M}^{2} P_{M}{ }^{2 / \alpha}} .
$$


(2) 覆盖面积限制. 微基站和宏基站提供的覆盖面积比值与热点区域面积和非热点区域面积的比 例相等, 即

$$
\gamma_{m}=\tilde{\gamma}_{m}=\frac{\lambda_{m} P_{m}^{2 / \alpha}}{\lambda_{M} P_{M}^{2 / \alpha}} .
$$

这两个限制条件, 再加上保证边缘用户最低信干噪比门限值的限制条件 $S_{\text {edge }} \geqslant \beta$, 建立了超蜂窝 网络宏基站发射功率、微基站发射功率、宏基站密度、微基站密度和业务不均匀性之间的制约联系.

本研究相关工作发表在文献 $[23,24]$ 上.

\section{2 基于用户群体行为的高能效传输控制方法}

利用所提出的用户聚集系数来描述超蜂窝网络用户行为, 然后基于用户行为和异构网络架构, 最 后提出在异构网络中通过基站协作进行组播和单播增补高能效的多媒体传输方案, 并找到使得能效值 达到最大的最优组播时窗和带宽分配策略.

为了满足用户的业务需求, 在仅有宏基站 MBS (macro base station) 覆盖的网络中灵活地放置低 功耗微基站 SBS (small base station). 当用户集中在某一区域产生大量的请求时, 该区域将增加微基 站部署数目. 图 9(a) 所示传统多媒体单播传输方案 (UMT, unicast multimedia transmission) 不考虑 用户聚集特性和基站协作, 即热点用户由微基站单播, 非热点用户由宏基站单播. 这会造成能量的极大 浪费. 在图 9(b) 中传统多媒体组播传输方案 (TMMT, traditional multicast transmission) 不考虑基站 协作, 对于热点用户, 由微基站进行组播并采用单播进行增补. 对于非热点用户, 由宏基站进行单播传 输. 根据用户的聚集特性和宏微基站协作传输, 图 9(c) 呈现了一种基站协作多媒体传输方案 (MTBSC, multimedia transmission trough base station cooperation). 宏基站支持热点区域组播和非热点区域单 播; 微基站支持对热点区域进行增补 (patching stream).

如图 10 所示, 比较基站协作的高能效传输方案 MTBSC 和传统方案 UMT 及 TMMT 可知, 在不 同用户聚集度下, 所提方案的能效比传统方案有显著提升. 当用户越聚集, 即 $h$ 越大, 所提方案的能效 值提升越明显. 因为当 $h$ 越大, 热点小区内的用户聚集程度越高, 即通过宏微基站协作加入组播流的 用户数越多. 相对于 TMMT 方案: 当 $h=0.55$ 时, 所提方案能效提升了 $36.7 \%$; 当 $h$ 提升到 0.81 时, 能 效值的提升达到了 $83.7 \%$. 因此所提 MTBSC 方案性能极大提升网络能效.

网络能效值随时间窗 $t_{\mathrm{w}}$ 变化的关系如图 11 所示, 能效是时窗长度的凹函数, 即存在 $t_{\mathrm{w}}^{\mathrm{opt}}$ 使得能 效值最大. 由图可知, 理论值的最优点和仿真值最优点很接近. 因此, 在实际多媒流传输中, 可以设定 合适的时窗值和带宽分配策略达到高能效传输.

本研究相关工作发表在文献 $[25]$ 上.

\section{3 高能效基站分级休眠策略}

实际网络中, 业务量在时间上存在周期性变化. 而当前基站部署普遍针对的是网络中的峰值业务 量, 这就导致在非峰值时间段, 基站会处于比较空闲的状态, 降低了网络的效率. 另一方面, 由于用户 的行为特征, 业务在空间上的分布也会呈现出很明显的聚集特性.

针对不同的流量行为在时间和空间上的特征, 将基站休眠层次分为浅级休眠和深级休眠两级. 于 是基站存在 3 种可选工作状态:

(1) 当基站服务范围内存在业务请求时, 基站处于活跃状态, 正常进行数据传输.

(2) 当在基站连接有用户, 但并不需要进行数据传输时, 基站进行浅级休眠. 此时基站关闭数据传 输模块, 但保留与用户的连接及信令交互, 以及基站的业务覆盖范围.

630 


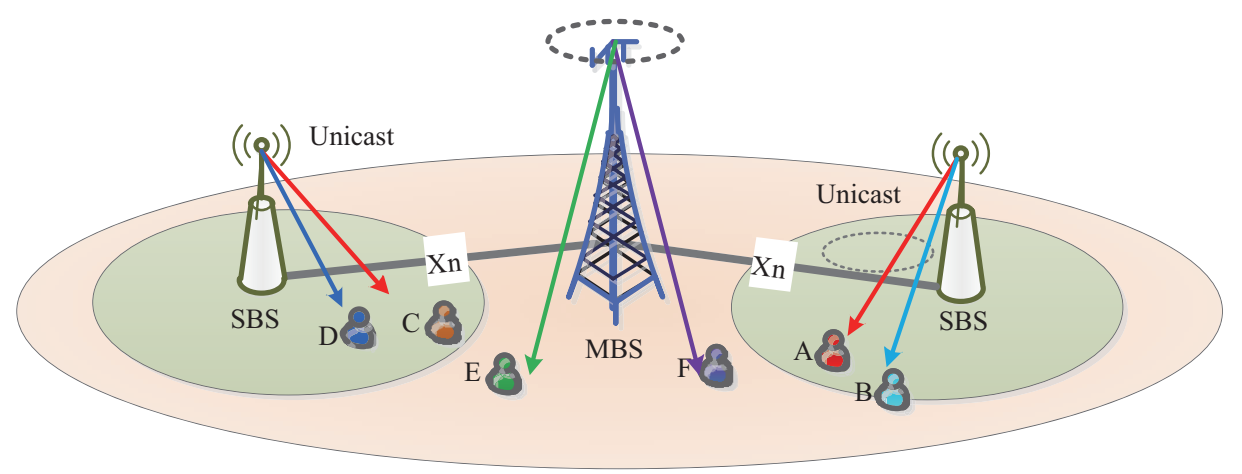

(a)

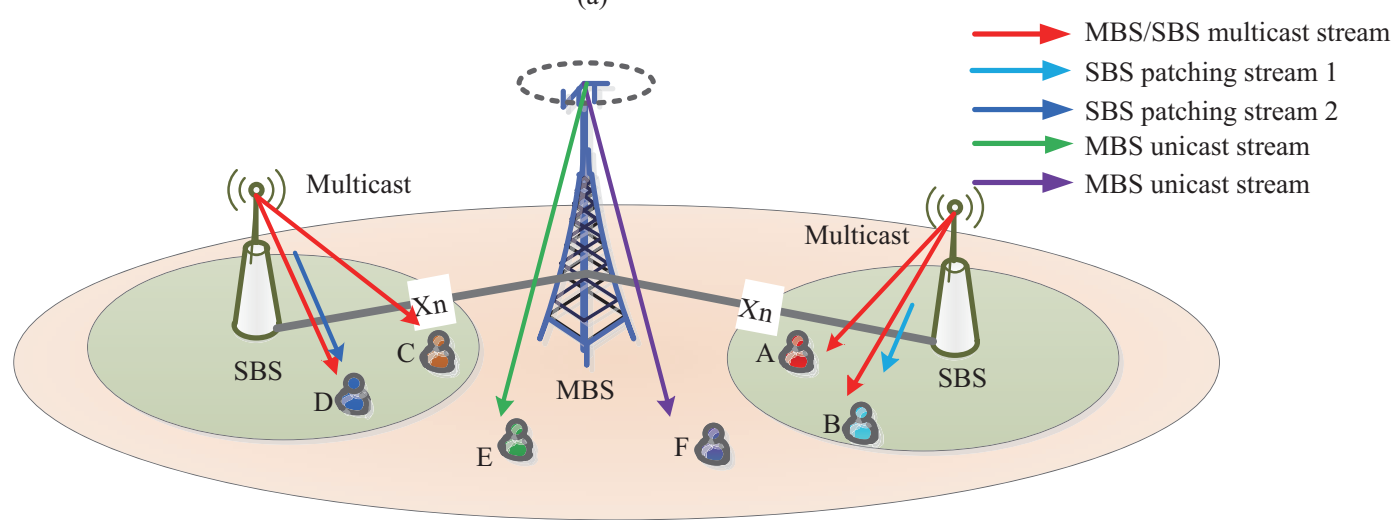

(b)

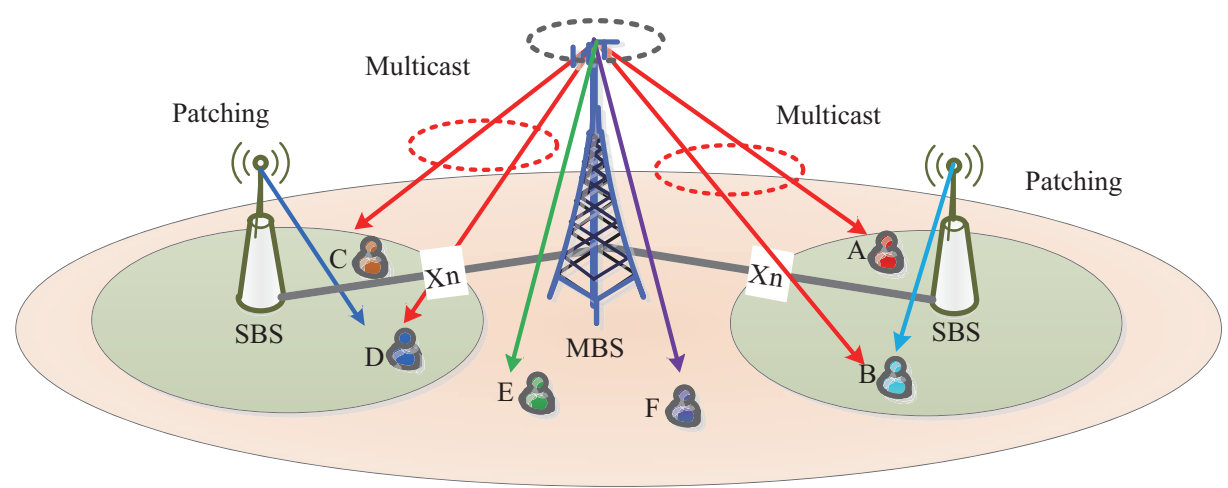

(c)

图 9 异构蜂窝网络下多媒体传输模式

Figure 9 Multimedia transmission schemes in heterogeneous cellular network. (a) Traditional unicast multimedia transmission (UMT); (b) traditional multicast multimedia transmission (TMMT); (c) proposed scheme: multimedia transmissions through base station cooperation exploiting user behavior (MTBSC)

(3) 当基站不连接用户时, 进行深级休眠. 此时基站关闭全部模块, 同时周围基站扩展覆盖范围以 保证业务服务无缝覆盖.

综合利用这两种休眠层次, 在保证业务服务质量满足一定需求的情况下, 达到网络能效的最优. 基 站双级休眠场景如图 12 所示.

假设基站服从 Poisson 分布, 密度为 $\lambda_{\mathrm{BS}}$, 每个基站的最大承载能力为 $U_{\max }$. 则当网络平均负载 


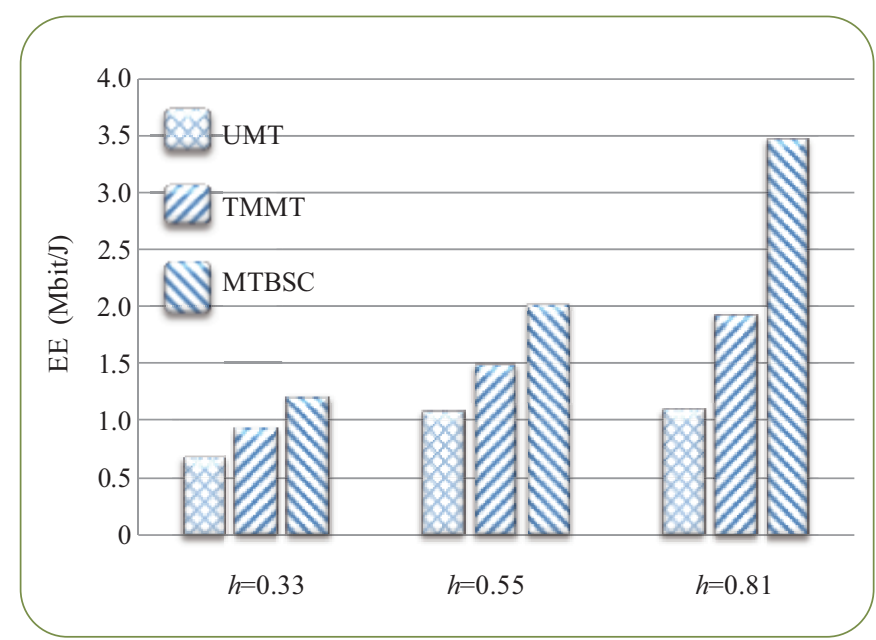

图 10 (网络版彩图) 不同用户聚集度系数下各方案的能效对比图

Figure 10 (Color online) The energy efficiency of the schemes in different user convergence coefficient

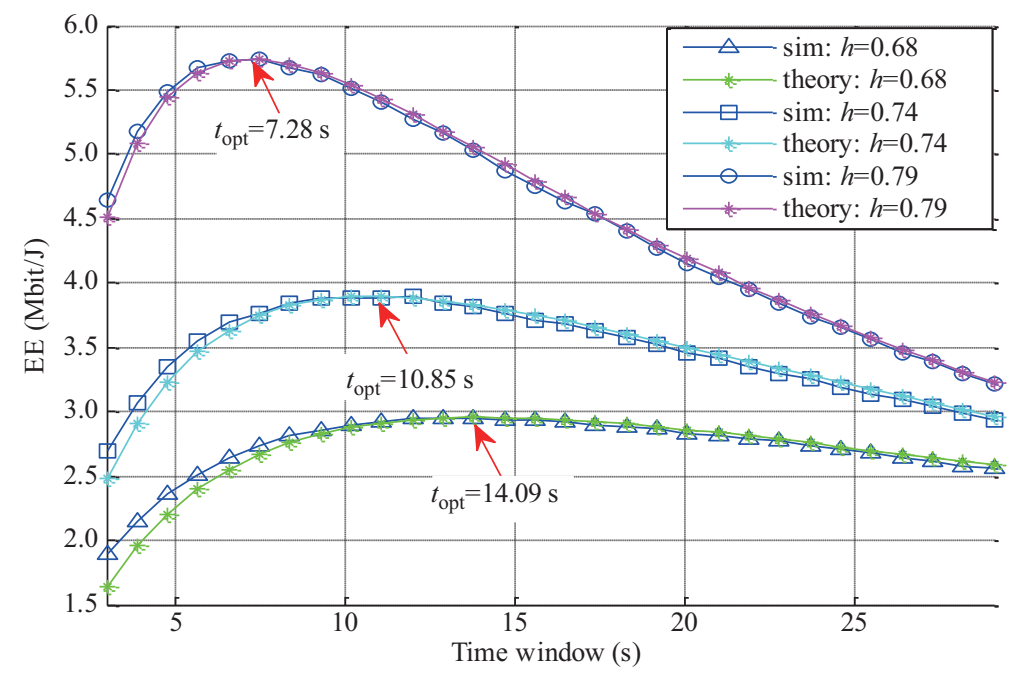

图 11 (网络版彩图) 不同用户聚集度系数下各方案的能效随时窗变化图

Figure 11 (Color online) Energy efficiency in different user convergence coefficient as time window is varied

为 $K$ 时, 网络中基站的深级休眠率为

$$
\operatorname{Pr}_{\mathrm{d}}(K)=\int_{0}^{K} \int_{u_{i}}^{\min \left(K, U_{\max }-u_{i}\right)} 1-\mathrm{e}^{-\lambda_{\mathrm{BS}} j\left(u_{i}, u_{j}\right)} \mathrm{d} u_{j} \mathrm{~d} u_{i},
$$

其中 $u_{i}$ 和 $u_{j}$ 为任意两相邻基站的实际负载.

此时网络中基站的浅级休眠率为

$$
\operatorname{Pr}_{1}=\left(1-\frac{K}{U_{\max }}\right)\left(1-\operatorname{Pr}_{\mathrm{d}}(K)\right) .
$$

基站处于活跃状态的概率为

$$
\operatorname{Pr}_{n}=\frac{K}{U_{\max }}\left(1-\operatorname{Pr}_{\mathrm{d}}(K)\right)
$$




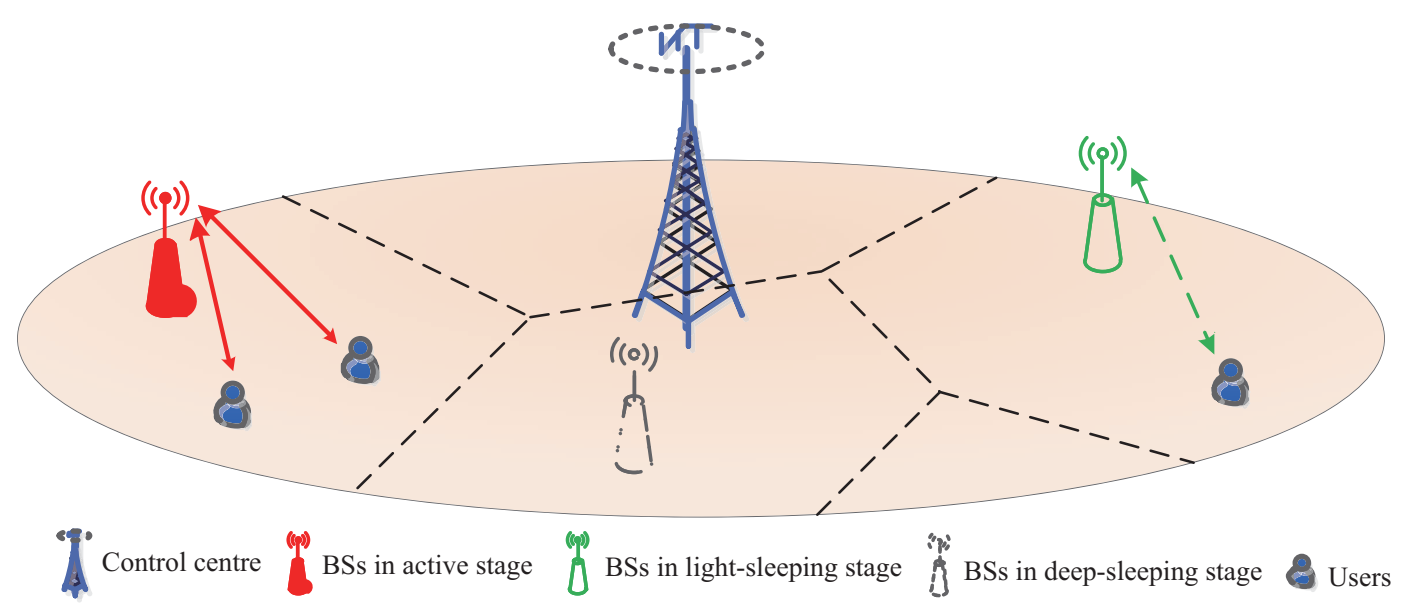

图 12 (网络版彩图) 基站双级休眠场景图

Figure 12 (Color online) The system model of two-stage base station sleeping scheme

从而得到采用所提基站双级休眠方案时, 网络中基站的平均功率

$$
P_{\text {avg }}(K)=\operatorname{Pr}_{n} \overline{P_{n}(K)}+\operatorname{Pr}_{1} \overline{P_{1}(K)}+\widetilde{E_{\text {sw }}(K)},
$$

以及网络中业务的平均时延

$$
D_{\text {avg }}=\operatorname{Pr}_{n} \overline{D_{n}}+\operatorname{Pr}_{1} \overline{D_{1}},
$$

其中 $\overline{P_{n}(K)}$ 和 $\overline{P_{1}(K)}$ 分别为基站在活跃状态和浅级休眠状态下的等效平均功率, $\widetilde{E_{\mathrm{sw}}(K)}$ 为等效状 态切换能耗. $\overline{D_{n}}$ 和 $\overline{D_{1}}$ 分别为基站在活跃状态和浅级休眠状态下的等效业务时延.

仿真表明所提基站双级休眠方案可以在业务时延增加约 $10 \%$ 的代价下，换取约 $150 \%$ 的节能效 果提升, 并且用户越聚集, 节能效果越好.

本研究相关工作发表在文献 $[26]$ 上.

\section{4 结论}

通过分别对南京、香港和苏州等城市的移动无线网络中业务数据采集和测量, 针对 IMT-2020 (5G) 所提的典型业务场景中的业务流量分布参数进行分析, 分别获取了基站流量在空域、时域和空 - 时 联合分布模型, 得到结论如下: 首先业务流量在空间符合 Log-normal 分布, 其参数与典型区域类型有 关; 其次用户数及其产生的业务量随着时间变化具有明显的规律性, 正弦叠加模型能够很好地反映出 现网实际业务量的变化情况; 最后得到能精准预测基站流量变化的空 - 时联合分布模型. 与实际数据 对比表明, 该模型准确度达到 $93 \%$ 以上. 基于对以上实际数据的分析研究, 给出了用户群体行为的定 义, 并使用经济学中的基尼系数对用户群体行为进行定量描述. 然后提出几种基于用户群体行为的高 能效资源配置方法、传输控制方法和基站分级休眠策略, 充分发掘了用户群体聚集行为在无线网络能 效提升方面的应用方法, 极大地提高了无线网络能效.

致谢感谢首席科学家牛志升教授对本文的指导. 


\section{参考文献}

1 Hohn N, Veitch D, Abry P. Does fractal scaling at the IP level depend on TCP flow arrival processes? In: Proceedings of the 2nd ACM SIGCOMM Workshop on Internet Measurment, Marseille, 2002. 63-68

2 Abry P, Veitch D, Flandrin P. Long-range dependence: revisiting aggregation with wavelets. J Time Ser Anal, 1998, 19: $253-266$

3 Feldmann A. Characteristics of TCP Connection Arrivals. In: Self-Similar Network Traffic and Performance Evaluation. Hoboken: John Wiley Sons Inc, 2002. 367-399

4 Floyd S, Paxson V. Difficulties in simulating the Internet. IEEE/ACM Trans Netw, 2001, 9: 392-403

5 Arfeen M A, Pawlikowski K, McNickle D, et al. The role of the weibull distribution in Internet traffic modeling. In: Proceedings of the 25th International Teletraffic Congress, Shanghai, 2013. 1-8

6 Adelantado F, Perez-Romero J, Sallent O. Nonuniform traffic distribution model in reverse link of multirate/multiservice WCDMA-based systems. IEEE Trans Veh Tech, 2007, 56: 2902-2914

7 Gotzner U, Gamst A, Rathgeber R. Spatial traffic distribution in cellular networks. In: Proceedings of the 48th IEEE Vehicular Technology Conference, Ottawa, 1998. 1994-1998

8 Ahn B, Yoon H, Cho J W. Joint deployment of macrocells and microcells over urban areas with spatially non-uniform traffic distributions. In: Proceedings of the 52nd IEEE Vehicular Technology Conference, Boston, 2000. 2634-2641

9 Ahn B, Yoon H, Cho J W. A design of macro-micro CDMA cellular overlays in the existing big urban areas. IEEE J Sel Area Commun, 2001, 19: 2094-2104

10 Tutschku K, Tran-Gia P. Spatial traffic estimation and characterization for mobile communication network design. IEEE J Sel Area Commun, 1998, 16: 804-811

11 Chandrasekhar V, Andrews J G, Gatherer A. Femtocell networks: a survey. IEEE Commun Mag, 2008, 46: 59-67

12 Ashraf I, Boccardi F, Ho L. SLEEP mode techniques for small cell deployments. IEEE Commun Mag, 2011, 49: 72-79

13 Hoydis J, Kobayashi M, Debbah M. Green small-cell networks. IEEE Veh Tech Mag, 2011, 6: 37-43

14 Quek T Q S, Cheung W C, Kountouris M. Energy efficiency analysis of two-tier heterogeneous networks. In: Proceedings of the 17th European Wireless 2011-Sustainable Wireless Technologies, Vienna, 2011. 1-5

15 Chandrasekhar V, Andrews J G. Spectrum allocation in tiered cellular networks. IEEE Trans Commun, 2009, 57: 3059-3068

16 Cheung W C, Quek T Q S, Kountouris M. Throughput optimization, spectrum sharing, and femtocell access in two-tier femtocell networks. IEEE J Sel Area Commun, 2012, 30: 561-574

17 Xia P, Chandrasekhar V, Andrews J G. Open vs. closed access femtocells in the uplink. IEEE Trans Wirel Commun, 2010, 9: 3798-3809

18 Chandrasekhar V, Andrews J G, Muharemovic T, et al. Power control in two-tier femtocell networks. IEEE Trans Wirel Commun, 2009, 8: 4316-4328

19 Ngo D T, Le L B, Le-Ngoc T, et al. Distributed interference management in two-tier CDMA femtocell networks. IEEE Trans Wirel Commun, 2012, 11: 979-989

20 Zhang J X, Wang W B, Zhang X, et al. Base stations from current mobile cellular networks: measurement, spatial modeling and analysis. In: Proceedings of the IEEE Wireless Communications and Networking Conference Workshops, Shanghai, 2013. 1-5

21 Wang S, Zhang X, Zhang J X, et al. An approach for spatial-temporal traffic modeling in mobile cellular networks. In: Proceedings of the 27th International Teletraffic Congress, Ghent, 2015. 203-209

22 Zhang X, Zhang Y, Yu R, et al. Enhancing spectral-energy efficiency for LTE-advanced heterogeneous networks: a users social pattern perspective. IEEE Wirel Commun, 2014, 21: 10-17

23 Huang Y, Zhang X, Zhang J X, et al. Energy efficient design in heterogeneous cellular networks based on large-scale user behavior constraints. IEEE Trans Wirel Commun, 2014, 13: 4746-4757

24 Huang Y, Zhang X, Wang W B, et al. Hybrid bandwidth allocation for energy saving based on large-scale user behavior in heterogeneous networks. In: Proceedings of the 9th International Conference on Communications and Networking 
in China, Maoming, 2014. 393-397

25 Zhang X, Yu R, Zhang Y, et al. Energy-efficient multimedia transmissions through base station cooperation over heterogeneous cellular networks exploiting user behavior. IEEE Wirel Commun, 2014, 21: 54-61

26 Yang J W, Zhang X, Wang W. Two-stage base station sleeping scheme for green cellular networks. J Commun Netw, 2016, 18: 600-609

\title{
The model of large-scale mobile user behavior and energy efficiency resource allocation method
}

\author{
Kun YANG ${ }^{1}$, Xing ZHANG ${ }^{*}$, Juwo YANG ${ }^{1}$, Shuo WANG ${ }^{1}$, Chengli MEI $^{2} \&$ Wenbo WANG $^{1}$ \\ 1. Key Laboratory of Universal Wireless Communications, Ministry of Education, Beijing University of Posts \\ and Telecommunications, Beijing 100876, China; \\ 2. Technology Innovation Center, China Telecom Corporation Limited, Beijing 100032, China \\ * Corresponding author. E-mail: hszhang@bupt.edu.cn
}

\begin{abstract}
For the existence of user social patterns, the traffic characteristics and user behavior in complex cellular mobile network show clustering behavior regularity characterized by groups in multi-dimensional distributions, such as time, space, and content. Previous static, island-like network resource allocation methods lead to a massive waste of network resources. Thus, the use of user behavior characteristics can have a huge impact on energy efficiency and resource utilization. Based on data collection and measurements from real cellular mobile communication systems, we analyze the behavior of user group aggregation in spatial, time, and other dimensions, to obtain traffic distributions across base stations in the space, time, and spatio-temporal domains. The study shows that traffic distribution in the space domain conforms to a Log-normal distribution and that its parameters are related to typical region types. The number of users and the amount of traffic they produce show regularity over time, and the Sine superposition model can reflect changes in the traffic volumes in a network. Next, through joint analysis of the space and time domains, we obtain a spatio-temporal joint distribution model that can accurately predict changes in base station traffic. When compared with real data, it is shown that the accuracy of the model reaches over $93 \%$. In order to characterize user group clustering more clearly, we use the Gini coefficient to present a mathematical definition and quantitative description of user group behavior. Finally, based on the proposed spatio-temporal model and user group behavior model, we propose several energy-efficient wireless network resource allocation methods, transmission control methods, and a base station hierarchical sleep strategy for exploring new approaches using user group behavior regularity to improve wireless network energy efficiency.
\end{abstract}

Keywords user behavior, resource allocation, energy efficiency, mobile cellular network 


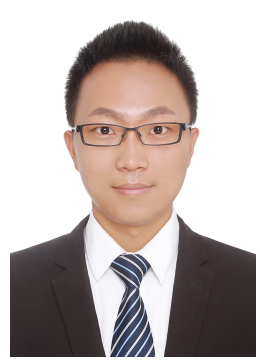

Kun YANG was born in 1987. He received B.E. and M.E. degrees from Shandong Normal University in 2010 and 2013, respectively. $\mathrm{He}$ is currently pursuing a Ph.D. degree at the School of Information and Communication Engineering, Beijing University of Posts and Telecommunications (BUPT), China. His research interests include green communications, 5G wireless communications and networks, small cell enhancement technologies, and cooperative communications.

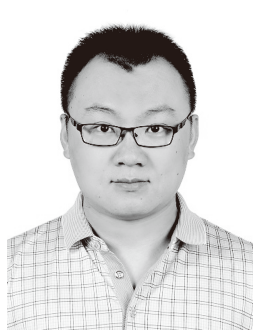

Juwo YANG was born in 1991. He received his B.S. degree from the Beijing University of Posts and Telecommunications (BUPT), Beijing, China in 2012. He is currently pursuing a Ph.D. at BUPT. His current research interests include green communication, 5G network architecture, and Internet of things technologies.

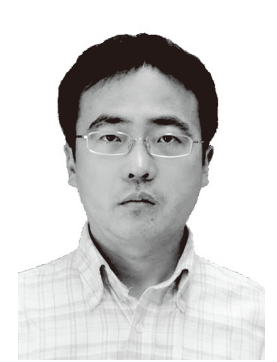

Xing ZHANG is full professor at the School of Information and Communications Engineering, Beijing University of Posts and Telecommunications (BUPT), China. His research interests include $5 \mathrm{G}$ wireless communications and networks, green communications, cognitive radio and cooperative communications, big data, and Internet of things.

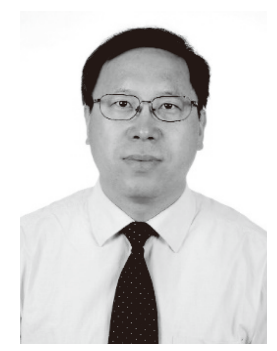

Wenbo WANG received his B.S. M.S., and Ph.D. degrees from the Beijing University of Posts and Telecommunications (BUPT) in 1986, 1989, and 1992, respectively. He is currently a professor and the executive dean at the Graduate School of BUPT. He is also deputy director of the Key Laboratory of Universal Wireless Communication, supported by the Ministry of Education. His current research interests include radio transmission technology, wireless network theory, and wireless signal processing. 